\title{
Fundamental Study on Flow Deflector to Improve Diffuser Performance
}

\author{
Yukai JIN $^{1^{*}}$, Junki YOSHIDA ${ }^{1 *}$, Takashi NOGUCHI ${ }^{1 *}$, Katsuya HIRATA ${ }^{1 *}$ \\ ${ }^{1}$ Doshisha University, Tataramiyakodani1-3, Kyotanabe City, Kyoto fu, Japan \\ ctwd0532@mail4.doshisha.ac.jp
}

\section{Introduction}

Diffusers of diverging pipes/ducts are extensively used in many industrial aspects. In consequence of various studies, diffuser works most efficiently at the expanding angle of 5 to 10 degree. However, in the automobile catalytic converter, an inefficient design of diffusers with large expanding angle under spatial and cost limitation has been designed. We have proposed a flow deflector inside the diffuser-part of an automobile catalytic converter, in order to reduce energy loss and to improve thermal uniformity. In this study, using conical and two-dimensional diffusers, we investigated experimentally the influence of the downstream substrate on the efficiency. In addition, we calculated the flow using OpenFOAM and compared it with the experimental results.

\section{Methods}

Figure 1 shows the conical diffuser with flow deflector model respectively. The present experimental apparatus is the same as our previous studies ${ }^{(1-3)}$.

Computation was carried out using open-source software OpenFOAM version 2.3.0. The overview of numerical set-up is summarized in Table 1.

\section{Results and Discussion}

\subsection{Pressure-loss coefficient from experiment}

Figure 2 shows the pressure-loss coefficient $\zeta$ of the conical diffuser plotted against $R e$. For reference, the figure also shows previous data of $\zeta^{(4)}$. The former concerns the ordinary-diffuser case with small $\theta$ s.

We can confirm that the influence of the flow deflector is remarkable; at $R e \gtrsim 4 \times 10^{4}$, the reduction on $\zeta$ is more than $20 \%$ ref even in the ordinary-diffuser case, as well as the downstream-substrate case ${ }^{(3)}$.

\subsection{Computation}

Figure 3 shows a typical visualized flow field obtained by computation, namely, time-averaged velocity vectors inside the diffuser with the flow deflector in the ordinary-diffuser case, on the center plane, at $R e=5.0 \times 10^{4}$. It is confirmed that the timeaveraged flow field is mostly axi-symmetric throughout the diffuser. We can see a strong annular flow whithout separation near the walls of the diffuser, in addition to the main flow.

\section{Concluding Remarks}

It is revealed that the flow deflector is effective for diffusers as well as catalytic converters.

\section{Reference}

(1) Hirata., et al., Proceedings IMechE, Part D: Journal of Automobile Engineering, 222-3, (2008) 455-467.
(2) Inoue., et al., Proceedings of International Workshop on Environment \& Engineering, 306, (2014) 1-6.

(3) Hirata., et al., JSME Mechanical Engineering Journal, 4-6, (2017) 1-17.

(4) Rend., et al., International Journal of Heat and Mass Transfer, Vol. 61, No. 1 (2013), pp. 56-61.

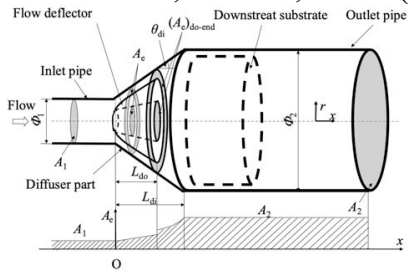

(a) Overview

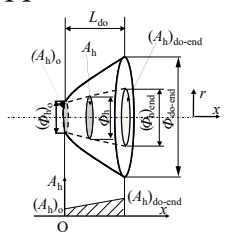

(b) Details of a solid-dome flow deflector
Fig. 1 Model: a conical diffuser with a flow deflector.

Table 1 Numerical set-up

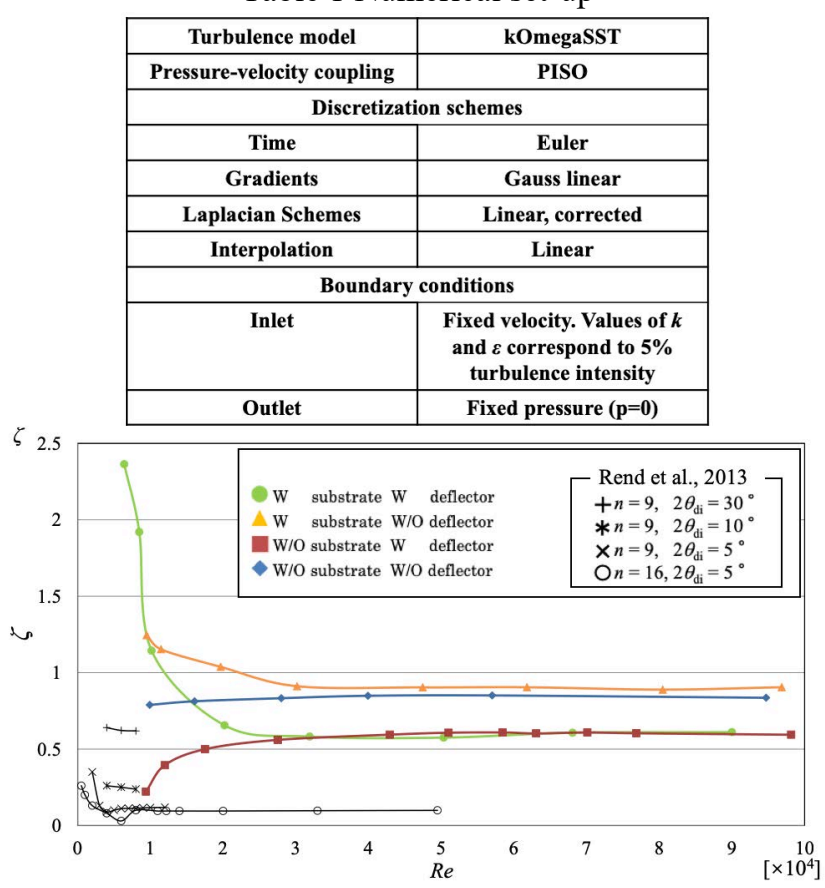

Fig. 2 Pressure-loss coefficient $\zeta$ of a conical diffuser versus $R e$

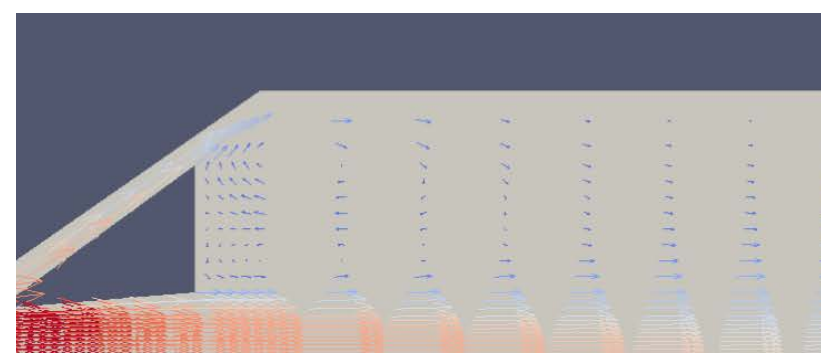

Fig. 3 Velocity vectors by computation 